\title{
Tomonaga-Luttinger parameters for quantum wires
}

\author{
Wolfgang Häusler ${ }^{1,2,3}$, Lars Kecke ${ }^{2}$, and A. H. MacDonald ${ }^{1,4}$ \\ 1 Department of Physics, Indiana University, 701 E Third Street, Swain Hall-West 117, Bloomington, IN 47405, U.S.A. \\ ${ }^{2}$ I. Institut für Theoretische Physik der Universität Hamburg, Jungiusstr. 9, D-20355 Hamburg, Germany \\ ${ }^{3}$ Fakultät für Physik, Albert-Ludwigs-Universität Freiburg, D-79104 Freiburg, Germany \\ ${ }^{4}$ Department of Physics, University of Texas at Austin, Austin TX, 78712, U.S.A.
}

\begin{abstract}
The low-energy properties of a homogeneous one-dimensional electron system are completely specified by two Tomonaga-Luttinger parameters $K_{\rho}$ and $v_{\sigma}$. In this paper we discuss microscopic estimates of the values of these parameters in semiconductor quantum wires that exploit their relationship to thermodynamic properties of the quantum wire. Motivated by the recognized similarity between correlations in the ground state of a one-dimensional electron liquid and correlations in a Wigner crystal, we evaluate these thermodynamic quantities in a self-consistent Hartree-Fock approximation. According to our calculations, the Hartree-Fock approximation ground state is a Wigner crystal at all electron densities and has antiferromagnetic order that gradually evolves from spin-density-wave to localized in character as the density is lowered. Our results for $K_{\rho}$ are in good agreement with weak-coupling perturbative estimates $K_{\rho}^{\text {pert }}$ at high densities, but deviate strongly at low densities, especially when the electron-electron interaction is screened at long distances. $K_{\rho}^{\text {pert }} \sim n^{1 / 2}$ vanishes at small carrier density $n$ whereas we conjecture that $K_{\rho} \rightarrow 1 / 2$ when $n \rightarrow 0$, implying that $K_{\rho}$ should pass through a minimum at an intermediate density. Observation of such a non-monotonic dependence on particle density would allow to measure the range of the microscopic interaction to be determined. In the spin sector we find that the spin velocity decreases with increasing interaction strength or decreasing $n$. Strong correlation effects make it difficult to obtain fully consistent estimates of $v_{\sigma}$ from Hartree-Fock calculations. We conjecture that $v_{\sigma} / v_{\mathrm{F}} \propto n / V_{0}$ in the limit $n \rightarrow 0$ where $V_{0}$ is the interaction strength.
\end{abstract}

PACS : 71.10.Pm, 71.10.-w, 71.10.Hf, 71.45.Lr

\section{INTRODUCTION AND OVERVIEW}

It has been known for some time that one-dimensional (1D) metals are different from their higher dimensional Fermi-liquid cousins 1 . It is generally believed that at low energies and long wavelengths one dimensional electron systems can, under very general circumstances, be described as 'Tomonaga-Luttinger (TL) liquids, although it has nearly always been difficult to provide incontrovertible experimental evidence. Interestin TL liquids has been heightened in recent years by new physical realizations, including quantum Hall edge systems $\mathrm{A}$. and semiconductor quantum wires $\mathrm{S}$ in particular. Like Fermi liquid theory, TL theory can be used to relate lowtemperature, low-frequency, long-wavelength properties to a small number of parameters in which the microscopic physics of particular systems is encoded. For example, TL thegry predicts that for continuous-one-channel quantum wires the quantized conductance is renormalized by the factor $10 K_{\rho}$ at frequencies larger than $11 v_{\mathrm{F}} / L$ ( $L$ is the wire length and $v_{\mathrm{F}}$ the Fermi velocity). Surprisingly, low-energy orthogonality catastrophes lead to spectral functions that follow power laws 12 . prefactor\$13. 14 associated with the presence of marginal operators like back scattering in spin sector) non-universal power laws specified by TL theory parameters are also predicted for the behavior of correlation functions at distances much larger than the spatial range of interactions. (The strictly infinite range Coulomb interaction case requires special considerations.15.66). Microscopic theory still has an important role at low energies however, in estimating the values of these parameters. This is especially important because the distinction between Fermi liquids and Luttinger liquids on the basis of a set of experimental data over a limited temperature or energy range is sometimes subtle, and the range of energies over which TL behavior is expected is often not accurately known. Approximate values of expected TL parameters can play a role in determining whether or not an experimental result reflects TL behavior $\mathrm{E}$. In addition, as this approximate calculation shows, the problem of understanding the value of the two independent TL parameters of a homogeneous one-dimensional electron system is a challenging many-body problem that is interesting in its how right.

Four TL parameters characterize the low energy properties of interacting spinful electrons moving in one channel. For the charge $(\nu=\rho)$ or spin $(\nu=\sigma)$ sector, the parameter $K_{\nu}$ fixes the exponents for most of the power laws and $v_{\nu}$ is the velocity of the long wave length excitations. Symmetries in the charge or in the spin sector reduce the number of independent parameters in the case of a one-dimensional electron gas system : spin rotation invariance enforces ${ }^{17}$ $K_{\sigma}=1$ while Galilean invariance implies that $18 v_{\rho}=v_{\mathrm{F}} / K_{\rho}$. The later identity does not apply, for example, in 
lattice models since it requires continuous translational invariance; in that case $v_{\rho}$ and $K_{\rho}$ must be determined independently. This leaves $K_{\rho}$ and $v_{\sigma}$ as the only two independent TL parameters for single-channel semiconductor quantum wires since they can be accurately described by a continuum envelope function approximation.

In Fermi liquids a traditional and successful strategy has separated the phenomenological application of Fermi liquid theory from the microscopic evaluation of its parameters. To date most theoretical TL activity has focused on phenomenological applications; confident interpretation of experiments will require reliable microscopic estimates of the theory's parameters for the various physical systems of current interest. The evaluation of Fermi liquid parameters in two and three dimensional metals is one of the classic early topics in many electron physics, with considerable recent progress coming from quantum Monte Carlo calculations 19 . Still, useful physical insight and reasonable accuracy has resulted from less computationally cumbersome approaches. In this paper we discuss what can and cannot be learned about the values of TL parameters in semiconductor quantum wires and the physics of their dependencies on system geometry using unrestricted Hartree-Fock estimates of ground state energies. The Hartree-Fock approximation can yield very reliable estimates to the boundary exponent 20 describing tunneling into the end of quantum wires and, as a microscopic approach, gives information about quantities not reliably accessible in the TL formalism, including absolute values for the prefactors of power laws.

For non-interacting electrons the TL parameter $K_{\mathrm{m}}=1$. With repulsive interactions its value should decrease and go to zero in the limit of very strong or long ranging 16 interactions. For microscopic interaction potential $V\left(x-x^{\prime}\right)$ the formula

$$
K_{\rho}^{-1}=\sqrt{1+4 m V(k=0) / \pi^{2} n}
$$

is commonly used in the literature. It depends on the carrier density $n$, the effective mass $m$ and the $k=0$ Fourier component of the interaction

$$
V(k)=\int \mathrm{d} x V(x) \cos k x .
$$

Relation (11) can be motivated by lowest order perturbation theory, or by the random phase approximation 21 , though it misses the Fock contribution for spinful electrons in 1D. Any naive higher order perturbative contribution is divergent only the infinite subsums that are conveniently captured using a perturbative renormalization approach are finite22). Eq. (1) completely ignores the renormalizing influence of short wave length modes in determining the actual values of the effective interaction. Higher order perturbative renormalization group calculations demonstrate how the interaction parameters are coupled and renormalize as short wave length contributions are integrated out23. One important example is back scattering, across the Fermi line, of opposite spins Fermions, the so called $g_{1}-$ process, that spoils the separate conservation of the number of left and right moving particles of a given spin and therewith is not included in the TL model. This interaction, which is finite in leading order perturbation theory, scales to zero during renormalization, restoring $\mathrm{SU}(2)$ spin rotation symmetry and the validity of the TL at low energies 4 . Even for a model of spinless electrons, the parameters will rescale at low energies, reflecting other irrelevant operators that are omitted in the TL model such as non-linear dispersion of the kinetic energy in the microscopic Hamiltonian 3 . As a consequence Eq. (1) cannot be used to estimate $K_{\rho}$ when interactions are strong.

How interactions influence the spin sector is even less certain. According to textbook knowledge 25 the spin velocity would not be altered by interaction forces that act only on spatial coordinates and thus not in the spin sector. Other work 15 includes exchange contributions in the Bose form of the Hamiltonian in a way violating the SU(2) invariance property, $K_{\sigma}=1$. On the other hand, changes in $v_{\sigma} / v_{\mathrm{F}}$ are quite crucial to various physical properties. It influences for instance the magnetic susceptibility, the $g$-factor, and_ppin transport properties. The latter are particularly important for potential one-dimensional spintronic devicese6. In one-dimensional channels27 for example the spin conductance 28, and Rashba precession in the presence of spin orbit coupling29, depend on $v_{\sigma}$. Most directly $v_{\sigma}$ can be measured by inelastic Raman scattering in the 'depolarized' configuration with perpendicularly polarized incident and outgoing light 30131 .

To date relations between the microscopic electron-electron interaction and resulting Tf parameters have been established for models_of primarily theoretical interest, such as the Kondo Lattice Model32, the Hubbard model $\mathbf{3 3}$, and the $t-J$-model. 34 For the latter two models the ground state energies are known exactly, either analytically in certain limiting cases or by solving the Bethe-Ansatz equations numerically. For these repulsire short range interaction models $K_{\rho}$ is found to be confined to the range $1 / 2 \leq K_{\rho} \leq 1$ and it has been argued 33 in the limits of either infinite interaction strength or vanishing particle density are equivalent to non-interacting spinless Fermions with $k_{\mathrm{F}}$ being replaced by $2 k_{\mathrm{F}}$ so that $K_{\rho} \rightarrow 1 / 2$ in either of these limits in order to recover the correct asymptotic decay of the density-density correlation function. For the $t-J$-model, TL parameters away from the super-symmetric point $(J / t=2)$ have been obtained by using ground state energies from exact diagonalization caleplations 35 . The Sutherland model for spinless Fermions, where $V(x)=\lambda / x^{2}$, has proven to be a TL at low energies $\$$. The asymptotic decay of 
its one-particle Greens function implies that $K_{\rho}=2 /(1+\sqrt{1+2 \lambda})$ with $1 \geq K_{\rho} \geq 0$ for repulsive interactions, $\lambda>0$. The compressibility of this system is proportional to $K_{\rho}^{2}$ and satisfies Eq. (3) below. For quantum wires with long but finite range Coulomb interactions the values of the TL parameters have been determined previously by extensive quantum Monte Carlo calculations37. However, the limits on the number of particles and lattice points in real space for which these calculations can be carried out in a reasonable time places limits on the range of particle densities over which accurate Monte Carlo results can be obtained. In particular the low-density regime where interactions are strongest is difficult to reach. In this work we also exploit the thermodynamic relations between the uniform static compressibility $\kappa$ and the TL parameter in the charge sector. For quantum wires we have (cf. Refs. [3, 33])

$$
\frac{1}{\kappa} \equiv \frac{\partial^{2}\left(E_{0} / L\right)}{\partial n^{2}}=\frac{\pi}{2} \frac{v_{\rho}}{K_{\rho}}=\frac{\pi}{2} \frac{v_{\mathrm{F}}}{K_{\rho}^{2}},
$$

where the last equality uses Galilean invariance.

Density-density correlation function calculations performed within the TL model have been used ${ }^{5}$ to show that long range interactions $V(|x|) \sim|x|^{-a}$ put the one-dimensional Fermion system ground state into a Wigner crystal when $a<1$, irrespective of the strength of $V$. The Coulomb case, $a=1$, is marginal and density-density correlations decay extremely slowly, slower than any power law at large distances. This observation suggests that the ground state energy $E_{0}$ and therefore any $T=0$ thermodynamic property should be accurately estimated by the unrestricted Hartree-Fock approximation, for which the ground state is a Wigner crystal. At high densities it is known 25 that the Hartree-Fock (HF) approximation reproduces the leading order perturbative RG result for $K_{\rho}$. Since it spontaneously breaks translational symmetry, the HF approximation correlation functions have infinite range. The tails of the correlation functions are therefore given slightly incorrectly. Much more important for the energy however, is the accurate estimate of the magnitude of the short-distance correlation function oscillations, illustrated in Fig. 1. The dominant periods for charge and spin densities are in agreement with TL model calculations, which are however, not able to estimate the amplitude of the oscillations. In the real correlation function, these oscillations are multiplied by an envelope function whose decay properties are inaccessible to Hartree-Fock theory but can be calculated from the TL theory. The accuracy of self-consistent Hartree-Fock energy estimates, particularly at low-densities, has been previously been established in other interacting electron systems.38

We compare our results for ground state energies and compressibilities $\kappa$ with estimates obtained within the harmonic approximation to the classical Wigner crystal, cf. (6.,7) and within perturbation theory $\mathrm{BS}^{\mathrm{S}}$. The perturbative expression (12) we use (see below) for the charge sector TL parameter $K_{\rho}$ turns out to be surprisingly accurate over a wide range of carrier densities including typical experimental ones. Only below densities corresponding to values $r_{\mathrm{s}}>1.3$ of the usual $r_{\mathrm{s}}$-parameter used to measure the interaction strength in metals (see below), do we find smaller $\kappa$ in the selfconsistent HF solution than given by Eq. (12) and (3). At even lower densities $n \lesssim 1 / R$, where $R$ is the long but finite range discussed below that we use for the electron-electron interaction, Eq. (12) predicts that $\kappa$ goes to zero whereas HF theory yields a finite limiting value, after $\kappa$ has passed through a minimum. This minimum is also reproduced by the harmonic approximation. In the latter approximation, however, $\kappa$ diverges as $n \rightarrow 0$. We shall give arguments supporting the conjecture that the true $K_{\rho}$ stays finite as $n \rightarrow 0$ and indeed that $K_{\rho} \rightarrow 1 / 2$ in this limit.

We also analyze the spin sector and compare different approaches in the attempt to determine the spin velocity $v_{\sigma}$. The simplest estimate is again low order perturbation theory for the magnetic susceptibility. Other estimates can be obtained by starting with the assumption that the system is close to an antiferromagnetic Heisenberg spin chain at low densities. It turns out that the estimates for $v_{\sigma}$ that follow from different plausible schemes differ substantially. Furthermore, at smaller particle densities they are not in good agreement with earlier QMC estimate 3 . Although we are able to conclude that $v_{\sigma}$ is small at low-densities, our results for the spin sector do not substantially improve on existing Monte Carlo results.

\section{MICROSCOPIC INTERACTION}

Now we discuss the form of realistic interactions $V$ along the $x$-direction of a quantum wire. At inter-particle separations $\left|x-x^{\prime}\right|$ larger than the diameter $d$ of the wire $V\left(\left|x-x^{\prime}\right| \gtrsim d\right)=e^{2} / \epsilon\left|x-x^{\prime}\right|$ will be of the Coulomb form, irrespective of the detailed shape of the transversal potential. If the material enclosing the wire is insulating with dielectric constant $\epsilon^{\prime}$, the Coulomb form still holds at larger distances, but with dielectric $\epsilon$ replaced by $\epsilon^{\prime}$. We assume here equal dielectric constants $\epsilon \sim \epsilon^{\prime}$, the case that applies to gated as well as in cleaved edge overgrowth structures 9 . Eventually, at an inter-particle separation exceeding the distance $R$ to the closest metallic system that is extended in the direction along the wire. This metallic screening can be supplied by carriers in nearby metallic gates, including those used to define the quantum wire. Assuming that the screening plane and the quantum wire are 
parallel $V\left(\left|x-x^{\prime}\right|>R\right) \sim 1 /\left|x-x^{\prime}\right|^{3}$ because of the formation of dipoles from image charges. The interaction (4) below accounts for this cut-off at large particle separations.

At distances $|x| \lesssim d$ shorter than the wire width, the precise transverse form of the electronic wave function influences $V(|x|)$. For example, in 2D hetero-structures all electrons share a common growth direction wave function. If this wave function is approximated by a harmonic oscillator with characteristic length $d$, and thickness in the 2nd confined direction is neglected, we have $41 V_{2 \mathrm{D}}(x)=\left(e^{2} / 2 \sqrt{\pi} \epsilon d\right) \mathrm{e}^{x^{2} / 8 d^{2}} K_{0}\left(x^{2} / 8 d^{2}\right)$ where $K_{0}$ denotes a Bessel function. It is more realistic to include finite thickness in both confined directions. For example, for 3D wires with circular cross sections, a model which might be appropriate for cleaved-edge-overgrowth systems, we have 22 $V_{3 \mathrm{D}}(x)=\left(e^{2} / \sqrt{2 / \pi} \epsilon d\right) \mathrm{e}^{x^{2} / 2 d^{2}} \operatorname{erfc}(x / \sqrt{2} d)$ again using harmonic oscillator ground states of widths $d / \sqrt{2}$, now for both of the transverse directions. Neither of these forms is smooth at $x=0$, an artifact of assuming factorized wave functions. At short distances, corresponding to high energies, the factorization assumption needs to be refined. It leads to the unphysically slow decay of the Fourier transforms $\hat{V}_{2 \mathrm{D}}(k)=\left(e^{2} / \epsilon v_{\mathrm{F}}\right) \mathrm{e}^{k^{2} d^{2} / 2} K_{0}\left(k^{2} d^{2} / 2\right)$, and $\hat{V}_{3 \mathrm{D}}(k)=$ $\left(e^{2} / \epsilon v_{\mathrm{F}}\right) \mathrm{e}^{k^{2} d^{2} / 2} E_{1}\left(k^{2} d^{2} / 2\right)$, as seen in Figure 2, $E_{1}$ is the exponential integral. Also included in Figure 2 2 is the more realistic form,

$$
\hat{V}(k)=\frac{2}{a_{\mathrm{B}} k_{\mathrm{F}}}\left[K_{0}(k d)-K_{0}\left(k \sqrt{d^{2}+4 R^{2}}\right)\right],
$$

which accounts for the image potential term from a remote screening plane separated by $R \gg d$. Its real space form is displayed in Eq. (A4). This interaction remains finite for $k \rightarrow 0$ and decreases more quickly for large $k$ and will be used in the present work. Here, and in the following we measure the interaction in units of the Fermi velocity so that $\hat{V}$ is dimensionless in Eq. (何. Its strength, in comparison with the kinetic energy, scales with the dimensionless parameter $r_{\mathrm{s}}:=1 /\left(2 n a_{\mathrm{B}}\right)=\pi /\left(4 k_{\mathrm{F}} a_{\mathrm{B}}\right)$ depending on density $n$ and the effective Bohr radius $a_{\mathrm{B}}$. In our calculations we assume $a_{\mathrm{B}}=2 d$.

Thus, two parameters

$$
R / d \quad \text { and } \quad k_{\mathrm{F}} d
$$

characterize the range and the strength of our model interaction (4), respectively. They both can be extracted quite reliably from experiment, $R$ from the sample lay out and $d$ from the energy $\sim 1 / m d^{2}$ of inter-subband excitations. Typical distances to metallic gates and typical wire width, as reported eg. in [ 8] and [ 9], correspond to values for $R / d$ ranging from 5 to 14 . Typical single wall carbon nanotube systems, on the other hand, would correspond to much larger $R / d$ values because of their extremely small diameters. Many of our calculations are for $R / d=5.66$ or 35.36. Note also that electron densities should be sufficiently low $\left(k_{\mathrm{F}} d<\sqrt{2}\right.$ within the parabolic approximation for the transverse confinement) to prevent occupation of the second subband.

\section{GROUND STATE ENERGY}

According to (3) we need to calculate ground state energies for different particle densities. In this work we employ the unrestricted Hartree-Fock approximation where details are described in Appendix A. Results of these calculations are included in subsequent Figures.

The close proximity to the Wigner crystal and the Bose character of all of the low energy excitations suggests comparing with the ground state energy density of the harmonic crystal in 1D,

$$
E_{0}^{\mathrm{wc}}=E_{0}^{\text {classical }}+\frac{1}{2} \int_{-k_{\mathrm{F}}}^{k_{\mathrm{F}}} \frac{\mathrm{d} k}{2 \pi} \omega(k)
$$

Here,

$$
E_{0}^{\text {classical }}=\frac{1}{2 L} \sum_{i \neq j} V\left(|i-j| \pi / 2 k_{\mathrm{F}}\right)
$$

is the classical contribution and the zero point energy follows from the phonon dispersion

$$
\omega^{2}(k)=\frac{1}{m} \sum_{j=1}^{\infty} V^{\prime \prime}\left(j \pi / 2 k_{\mathrm{F}}\right)\left(1-\cos \left(j k \pi / 2 k_{\mathrm{F}}\right)\right)
$$


of harmonic excitations. The primes denote derivatives w.r.t. the argument. Both, $E_{0}^{\text {classical }}$ and $E_{0}^{\text {wc }}$ provide rigorous lower bounds to the true ground state energy since the quartic term of the Coulomb interaction is positive when expanded in a power series and the Fermionic antisymmetry constraint, ignored by Eq. (6), increases the true Fermionic energy further. This latter observation remains true also for spin carrying electrons since spin cannot provide complete antisymmetry for symmetric spatial wave functions for more than two particles.

Figures 3 also include the lowest order perturbation theory estimate

$$
E_{0}^{\text {pert }}=\frac{v_{\mathrm{F}} k_{\mathrm{F}}^{2}}{3 \pi}+\frac{2 v_{\mathrm{F}} k_{\mathrm{F}}^{2}}{\pi^{2}} \hat{V}(0)-\frac{v_{\mathrm{F}}}{2 \pi^{2}} \int_{0}^{2 k_{\mathrm{F}}} \mathrm{d} k\left(2 k_{\mathrm{F}}-k\right) \hat{V}\left(\frac{k}{k_{\mathrm{F}}}\right),
$$

obtained by taking the Hamiltonian's expectation value in the non-interacting electron state, to obtain the positive Hartree, and the negative exchange contribution. The variational principle ensures that Eq. (9) is a rigorous upper bound to the ground state energy. The true ground state energy must lie between these two bounds.

The energy densities are plotted in dimensionless units:

$$
e_{0}\left(k_{\mathrm{F}}\right):=\frac{E_{0} / L}{k_{\mathrm{F}}^{3} / m}
$$

which has the value $1 / 3 \pi$ without interactions. The HF-energies $e_{0}^{\mathrm{HF}}$, seen in Figure 3 a for $R / d=5.66$ and in Figure $3 \mathrm{~b}$ for $R / d=35.36$, approach this value in the weakly interacting high density limit, $k_{\mathrm{F}} \rightarrow \infty$.

For densities above $k_{\mathrm{F}} d \gtrsim 0.5$, corresponding to $r_{\mathrm{s}} \lesssim 0.8, e_{0}^{\mathrm{HF}}$ agrees quite well with the perturbative estimate (9). This is despite the fact that the self consistent charge density modulation already shows significant amplitude in this regime as seen in Figure 6 below.

Below $k_{\mathrm{F}} d \lesssim 0.3\left(r_{\mathrm{s}} \gtrsim 1.3\right)$, the three approximations start to spread apart significantly. As $k_{\mathrm{F}} \rightarrow 0$ perturbation theory result diverges, $e_{0}^{\text {pert }} \sim 1 / k_{\mathrm{F}}$, while $e_{0}^{\text {wc }} \sim k_{\mathrm{F}}^{1 / 2}$ goes to zero. The mean field result $e_{0}^{\mathrm{HF}}$ approaches a finite value, a result which seems plausible since the zero point quantum fluctuation energy exceeds the classical interaction energy of the Wigner crystal at low densities for interactions decaying faster than $\sim 1 /\left|x-x^{\prime}\right|^{2}$ at large particle separations. We speculate that realistic quantum wires, which never have strictly infinite range interactions, always cross over into the hard sphere gases at sufficiently low densities. For this latter system it is known that the ground state energy approaches $e_{0} \rightarrow 4 / 3 \pi \approx 0.4244$ (cf. [ 3.43]), irrespective of the particle type, Fermionic or Bosonic, and irrespective of the particle's spin. The 'radius' of the hard spheres is unimportant when $k_{\mathrm{F}} \rightarrow 0$. Among the approximations discussed above $e_{0}^{\mathrm{HF}}$ is the only one that stays finite in this limit, though the limit it approaches is larger than $4 / 3 \pi$.

\section{TL PARAMETER $K_{\rho}$}

Figure日 shows

$$
1 / K_{\rho}=\left(\frac{\pi}{2}\left[k_{\mathrm{F}}^{2} e_{0}^{\prime \prime}\left(k_{\mathrm{F}}\right)+6\left(k_{\mathrm{F}} e_{0}^{\prime}\left(k_{\mathrm{F}}\right)+e_{0}\left(k_{\mathrm{F}}\right)\right)\right]\right)^{1 / 2}
$$

versus $k_{\mathrm{F}} d$ for $R / d=5.66$ (Figure 1 a) and for $R / d=35.36$ (Figure $1 \mathrm{~b}$ ). Eq. (11) follows from relation (3) together with (10); the primes again denote derivatives w.r.t. the arguments. Also included in these figures is the result from expression (11) and the perturbative estimate

$$
1 / K_{\rho}^{\text {pert }}=\left(1+\left(2 \hat{V}(k=0)-\hat{V}\left(k=2 k_{\mathrm{F}}\right)\right) / \pi\right)^{1 / 2},
$$

which follows from Eqs. (3) and (9)). Note that only this form, with the Fock term included, satisfies the physical requirement that spinless Fermions cannot feel contact interactions and that therefore $K_{\rho}$ equals unity for this model. Since the factor of two in (12) is absent in the spinless case, Eq. (12) indeed fulfills this Pauli principle requirement, unlike Eq. (11). Figure 1 also includes $1 / K_{\rho}^{\mathrm{cl}}$ and $1 / K_{\rho}^{\mathrm{wc}}$, calculated from the corresponding harmonic crystal energy estimates of Eqs. (7) and (6) using Eq. (3).

Over a wide range of densities, including the typical experimental regime, all of these approximations coarsely agree, though none of them can provide a rigorous bound on the exact compressibility or $K_{\rho}$. As for the ground state energies, the approximations start to deviate severely from one another at smaller densities, corresponding to $r_{\mathrm{s}} \gtrsim 1.5$. Both HF and harmonic estimates show non-monotonic behavior of $1 / K_{\rho}$ as a function of density, in agreement with recent quantum Monte Carlo 37 calculations. If, as we have conjectured, $e_{0}$ approaches a constant for $k_{\mathrm{F}} \rightarrow 0$, 


$$
K_{\rho}\left(k_{\mathrm{F}} \rightarrow 0\right)=\left(3 \pi e_{0}\left(k_{\mathrm{F}} \rightarrow 0\right)\right)^{-1 / 2} .
$$

Note that the HF compressibility approaches a constant in the low density limit. Conjecturing again that at mean particle separations exceeding the interaction range, $k_{\mathrm{F}} \ll \pi / 2 R$, the system crosses over into the hard core Bose gas with $e_{0} \rightarrow 4 / 3 \pi$ Eq. (13) would yield

$$
K_{\rho} \longrightarrow 1 / 2
$$

We note, for example, that the Hubbard model approaches (14) at small fillings, independent of the interaction strength $U$. The same holds true for the Fermi gas with contact repulsion3.44. It is an important observation that the limiting value $K_{\rho}^{\mathrm{HF}}\left(k_{\mathrm{F}} \rightarrow 0\right) \approx 029$ to 0.35 for $R / d=50 \ldots 8$ clearly exceeds $1 / 8$ which would be the limiting value for the extended Hubbard model 15 that has both on-site and near-neighbor interactions. On the other hand, as seen in Figure 1, the minimum value for $K_{\rho}$ at about $k_{\mathrm{F}} R \sim 1$ is considerably smaller than $1 / 2$, so that, contrary to the Hubbard model, the limit (14) would have to be approached from below with decreasing carrier densities in quantum wires.

Upon inspecting Figures 4 more closely a regime can be identified at densities somewhat above the maximum of $1 / K_{\rho}^{\mathrm{HF}}$ where $1 / K_{\rho}^{\mathrm{HF}}$ exceeds $1 / K_{\rho}^{\mathrm{pert}}$. As seen in Figure 6 , the relative increase in stiffness appears along with the occurrence of significant $4 k_{\mathrm{F}}-$ periodic contribution to the charge density modulation. Figure 6 shows the two lowest Fourier coefficients

$$
\varrho_{j} \equiv \varrho_{\uparrow}\left(q=j 2 k_{\mathrm{F}}\right)=(-1)^{j} \varrho_{\downarrow}\left(q=j 2 k_{\mathrm{F}}\right)=\varrho_{-j}
$$

for $j=1,2$ in units of the mean density $\varrho_{0}=2 k_{\mathrm{F}} / \pi$. In view of (15), which follows from Eq. (A7) of the Appendix, $4 k_{\mathrm{F}}$-periodic modulations of the charge density $\varrho_{\uparrow}(x)+\varrho_{\downarrow}(x)$ are given by the $j=2$ contribution in Figure 6 . The appearance of a substantial $j=2$ Fourier component, at $k_{\mathrm{F}} d \sim 0.5$, marks the crossover from spin-density-wave to Wigner crystal self-consistent solutions of the HF equations. A similar conclusion has been drawn from the extremely slow spatial decay of the density-density correlation function in the presence of long range interactions 15 and from recent quantum Monte Carlo studies 37 . With smaller $1 / R$ this regime of Wigner crystal-like states marked by

enhanced stiffness extends down to smaller densities and becomes more pronounced. The variation of $1 / K_{\rho}^{\mathrm{HF}}$ with $R$ is depicted in Figure 5 for the density $k_{\mathrm{F}} d=0.15$. At $k_{\mathrm{F}} R \gg 1$ all of the approximate estimates are consistent with the logarithmic increase $1 / K_{\rho} \sim \sqrt{\log R / d}$ suggested by perturbation theory. For $k_{\mathrm{F}} R \gg 1$, the electrostatic energy is so dominant that the energy and $K_{\rho}$ are relatively insensitive to correlations.

\section{SPIN SECTOR}

As mentioned already in the introduction, it is much more difficult to estimate how interactions influence the spin sector, and particularly its low energy TL parameter $v_{\sigma}$, than it is to estimate the charge sector parameter. In the model originally proposed by Luttinger ${ }^{2}$ with left and right going particles treated as distinguishable, the spin velocity is unrenormalized $46, v_{\sigma}=v_{\mathrm{F}}$, because the exchange term vanishes leaving magnetic properties of the system independent of interactions. For the Hubbard model, on the other hand, is known that the spin velocity 47 is dependent on interactions and particle density, $n$, vanishing like $n^{2}$ at small density $n$ for any finiteinteraction strength. The spin TL parameter is related to a thermodynamic quantity, the magnetic susceptibility, by 48

$$
\chi \equiv 4\left(\pi^{2} v_{\mathrm{F}} \partial_{m}^{2} e_{0}(m)\right)^{-1}=\frac{2 K_{\sigma}}{\pi v_{\sigma}}
$$

where $m=\left(n_{\uparrow}-n_{\downarrow}\right) / n$ is the magnetization per particle and $e_{0}$ is the dimensionless ground state energy density as defined in Eq. (10). Relation (16) actually holds for any interacting electron system in the single channel TL phase 4 . 17 . Evaluating $e_{0}(m)$ for the microscopic model perturbatively would give

$$
\tilde{v}_{\sigma} / v_{\mathrm{F}}=1-\hat{V}\left(2 k_{\mathrm{F}}\right) / \pi,
$$

using (16) and $K_{\sigma}=1$. Alternatively one also could impose a spin current $\ell=\left(n_{\mathrm{R} \uparrow}-n_{\mathrm{R} \downarrow}-n_{\mathrm{L} \uparrow}+n_{\mathrm{L} \downarrow}\right) / n$ per particle $\left(n_{\mathrm{R} / \mathrm{L} s}\right.$ are right/left moving densities of spin $\left.s\right)$ and measure the change in ground state energy

$$
\chi_{\ell} \equiv 4\left(\pi^{2} v_{\mathrm{F}} \partial_{\ell}^{2} e_{0}(\ell)\right)^{-1}=\frac{2}{\pi v_{\sigma} K_{\sigma}} .
$$


Perturbatively this gives an unchanged spin velocity, a result that simply reflects the fact that lowest order perturbation theory cannot describe drag effects 50 between the density fluctuations of opposite spins and thus leaves the system Galilean invariant in spin sector. Solving equations (16) and (18) for $K_{\sigma}$ yields $K_{\sigma}>1$ as a perturbative result for repulsive interactions which would contradict $\mathrm{SU}(2)$ invariance in a TL model. To enforce the SU(2) symmetry we can combine equations (16) and (18) and solve for $v_{\sigma}$ by eliminating $K_{\sigma}$. The result,

$$
v_{\sigma}^{\text {pert }}=\frac{2}{\pi}(\chi \chi \ell)^{-1 / 2}=v_{\mathrm{F}} \sqrt{1-\hat{V}\left(2 k_{\mathrm{F}}\right) / \pi}
$$

indeed agrees clearly better with the QMC data 37 than (17). Eq. (19) is included in Figure 8. At $\hat{V}\left(2 k_{\mathrm{F}}\right)=\pi$ the perturbative estimate $v_{\sigma}^{\text {pert }}$ vanishes and for smaller $k_{\mathrm{F}}$ the Fock term in (19) favors a spin polarized ground state. This result contradicts very general arguments that guarantee a non-magnetic ground state for any non-singular pair interaction potential in one dimension 51 . The true spin velocity should stay positive and approach zero only at vanishing particle density.

That the extraction of spin velocities from HF calculations is less reliable than the extraction of charge TL parameters, is already clear because of the incorrectly broken spin-rotational invariance in the HF ground state In the HF spin-density wave state we evaluate the spin-susceptibility by polarizing spins along the quantization axes. We can consider only cases with rational ratios of the spin-up and spin-down carrier densities. Because the periods of the spin-up and spin-down density waves differ in these solutions, it is more convenient to use a real space basis, discretizing space $\psi\left(x=x_{i}\right) \rightarrow \psi(i)$ as described in Appendix B. Self consistent solutions are shown in Figure 7. At finite magnetization this structure contains now 'defects', reflecting the loss of the $4 k_{\mathrm{F}}$-periodic component in the charge density modulations 52 . At least $N=44$ electrons have been considered on $M=401$ grid points, the smaller particle densities are based on $N=84$ and $M=801$ to avoid lattice artifacts to a high accuracy. These sizes are clearly beyond what presently can be treated with numerical many body approaches, like quantum Monte Carlo, but pose no problem here. Spin velocities obtained from $E_{0}^{\mathrm{HF}}(m) / L$ by virtue of Eq. (16) are included in Figure \&. Below $k_{\mathrm{F}} d=0.2$ it is very difficult to extract positive spin velocities. We see that selfconsistency pushes the point of vanishing spin velocity and the (erroneous) transition into a ferromagnetic ground state down to smaller densities compared to the perturbative estimate in Eq. (19), but the transition still occurs.

An alternative attempt to estimate the spin velocity starts from the argument that the electron spin sector would evolve at low particle densities towards that of an antiferromagnetic Heisenberg spin chain, as suggested by the staggered spin density profile found in the mean field solution, Fig 1. This argument is also suggested by the pronounced antiferromagnetic correlations found in the TL liquid spin sector, particularly for long range interactions 15 , and in finite pieces of one-dimensional wires53. Antiferromagnetic spin chains are known to represent microscopic models, such as the Hubbard model, at low energies and have been intensively investigated for instance by employing manifestly $\mathrm{SU}(2)$ spin rotation invariant non-Abelian bosonization 1454.

In the antiferromagnetic Heisenberg chain spin excitations (magnons) move at velocity

$$
v_{\sigma}=\pi^{2} J / 4 k_{\mathrm{F}}
$$

where $J$ is the nearest neighbor coupling constant. One possibility to guess the magnitude of $J$ is to compare the HF estimates for the ground state energy $e_{0}^{\mathrm{HF}}$ of unpolarized electrons with the ground state energy $e_{0}^{\mathrm{pol}}$ of fully spin polarized electrons. For the antiferromagnetic Heisenberg chain this energy difference, $J(1+\ln 2)$ per spin, 55 is known exactly. Equating the energy differences gives

$$
J=\frac{\pi}{1+\ln 2} \frac{v_{\mathrm{F}} k_{\mathrm{F}}}{2}\left(e_{0}^{\mathrm{pol}}-e_{0}^{\mathrm{HF}}\right)
$$

from which

$$
v_{\sigma}^{\mathrm{J}} / v_{\mathrm{F}}=\pi^{3}\left(e_{0}^{\mathrm{pol}}-e_{0}^{\mathrm{HF}}\right) / 8(1+\ln 2)
$$

follows. Eq. (21) is included in Figure 8. The transition into the spin polarized ground state occurs at $k_{\mathrm{F}} d=0.19$ $\left(r_{\mathrm{s}}=2.07\right)$ for $R / d=5.66$. Equation (20) can be checked for consistency in the non-interacting limit, $k_{\mathrm{F}} \rightarrow \infty$, where $v_{\sigma} \rightarrow v_{\mathrm{F}}$. Magnons would move at velocity $v_{\mathrm{F}}$ if $J=4 v_{\mathrm{F}} k_{\mathrm{F}} / \pi^{2}=0.41 v_{\mathrm{F}} k_{\mathrm{F}}$. On the other hand, $\left(e_{0}^{\mathrm{pol}}-e_{0}^{\mathrm{HF}}\right) \rightarrow 1 / \pi$ in this limit so that (20) yields $J=0.30 v_{\mathrm{F}} k_{\mathrm{F}}$. In view of the fact that the weak interaction limit is poorly described by the antiferromagnetic spin chain this picture seems amazingly consistent.

Recently, Calmels and Gold have calculated magnetic susceptibilities of quantum wires 56 , though for a different microscopic interaction, using standard heuristic approximations from electron gas theory 5 . By virtue of Eq. (16) these data allow us to extract spin velocities $v_{\sigma}^{\mathrm{CG}}$ which turn out to be slightly larger than our $v_{\sigma}^{\mathrm{HF}}$ values. Note 
that the perturbative estimate shown in Figure 2 of Ref. [ 56] uses Eq. (17) while in Figure 8 Eq. (19) is included. Compared with (19) the data $v_{\sigma}^{\mathrm{CG}}$ are smaller than $v_{\sigma}^{\text {pert }}$ at large densities, like the Hartree-Fock data. This latter property does not agree with the behavior obtained using QMCBJ where $v_{\sigma}^{\mathrm{QMC}}$ instead exceeds $v_{\sigma}^{\text {pert }}$. We conclude that $\mathrm{HF}$ and other approximations of the mean field type can provide only a qualitative guideline to $v_{\sigma}$. All of these attempts, however, agree in predicting spin velocities that depend on the interaction and decrease with increasing interaction strength. This result calls attention to the frequent assumptions in the literature that interactions not explicitly depending on spin would leave $v_{\sigma}$ unchanged25. This result should show up in current experiments, such as those described in Ref. [9], where typical values for $k_{\mathrm{F}} d \approx 0.3$ are in the regime investigated here. As already pointed out in the introduction, this parameter should influence measurable quantities, such as the spin-splitting enhancement factor, Raman scattering in depolarized configuration 11 , spin transport properties 28 , and Rashba precession 29.

Let us now discuss the low density limit using our conjecture that quantum wires become equivalent to the on-site Hubbard model in the limit of small particle densities. Hubbard model (lattice constant $a$ ) parameters, $t=v_{\mathrm{F}} / 2 k_{\mathrm{F}} a^{2}$ and $U=\hat{V}(k=0) v_{\mathrm{F}} / a$, can be related to microscopic parameters by equating the effective mass and the Coulomb barrier for a twa electron exchange, $\hat{V}(k)$ is defined in Eq.(2). To leading order in $t / U$ the spin velocity of the Hubbard model $v_{\sigma}^{\mathrm{HM}}$ is 17

$$
v_{\sigma}^{\mathrm{HM}} \stackrel{U \rightarrow \infty}{\longrightarrow} \frac{2 \pi a t^{2}}{U}\left(1-\frac{\sin 4 k_{\mathrm{F}} a}{4 k_{\mathrm{F}} a}\right)
$$

so that

$$
\frac{v_{\sigma}^{\mathrm{HM}}}{v_{\mathrm{F}}}=4 \pi / 3 \hat{V}(k=0)
$$

for small $k_{\mathrm{F}} a$, where the lattice constant is irrelevant. Note that $\hat{V} \sim v_{\mathrm{F}}^{-1}$ and thus $v_{\sigma}^{\mathrm{HM}} \propto k_{\mathrm{F}}^{2}$. This result agrees with the strong interaction limit of the continuum version, the electron gas with repulsive contact interactions 44 . Restoring quantum wire parameters Eq. (22) translates into

$$
\frac{v_{\sigma}}{v_{\mathrm{F}}} \stackrel{k_{\mathrm{F}} \rightarrow 0}{\longrightarrow} \frac{2 \pi}{3} \frac{k_{\mathrm{F}} a_{\mathrm{B}}}{\ln (2 R / d)}
$$

for $R / d \gg 1$. The available QMC data are consistent with (23), though, as in the charge sector, they are not conclusive enough to really confirm the low density equivalence.

\section{SUMMARY AND DISCUSSION}

Many non-trivial theoretical predictions exist in the literature on various measurable low energy properties of single channel quantum wires that are based on the TL model which describes the non-Fermi liquid behavior of these systems. Much in the spirit of the Landau theory of Fermi liquids all these predictions depend only on few phenomenological parameters. In the case of quantum wires with only one subband occupied there is only one parameter per degree of freedom, $K_{\rho}$ for the charge sector and $v_{\sigma}$ for the spin sector. In the absence of interactions these parameters assume the values $K_{\rho}=1$ and $v_{\sigma}=v_{\mathrm{F}}$. In this work we have investigated how the microscopic pair potential $V\left(x-x^{\prime}\right)$ changes $K_{\rho}$ and $v_{\sigma}$. We have considered a realistic, tractable, and sufficiently general form for $V\left(x-x^{\prime}\right)$, Eq. ( $\mathrm{A} 4)$, that depends on the diameter $d$ of the quantum wire which is measurable through the subband energy, and the distance to the nearest metallic gates $R$ as given by the sample lay out. Our approach is to relate the two TL parameters to thermodynamic quantities which we estimate on the basis of self consistent, unrestricted Hartree-Fock (HF) approximations for the ground state energy.

In the charge sector this strategy is found to yield reasonably accurate results. At densities corresponding to $r_{\mathrm{s}} \lesssim 1.3$ we confirm applicability of the perturbative formula (12) for $K_{\rho}$. This regime includes most of the experiments based on semiconducting heterostructures 31 . At somewhat smaller densities Eq. (12) even overestimates $K_{\rho}$. In this regime we find enhanced stiffness compared to perturbation theory, signaling the close proximity of a Wigner-crystal state. For this reason quantitative corrections to Eq. (12) may arise when $R \ggg d$, for example in carbon nanotubes. In quantum wires fabricated on the basis of semiconducting hetero-structures with gates, the perturbative formula may be used even down to densities $2 k_{\mathrm{F}} / \pi \approx 1 / R$. The proximity to the Wigner-crystal state competes with the finite interaction range in these systems. Irrelevant or marginal operators in the microscopic Hamiltonian, such as non-linear single particle dispersion or backward scattering in the spin sector turn out to be unexpectedly inefficient to renormalize the TL parameters in the charge sector up to moderate interaction strengths. With decreasing density 
the values for $K_{\rho}$ clearly fall short of $1 / 8$ which is the minimum assumed by the extended Hubbard model including repulsions on neighboring lattice sites and often is considered to emulate models of finite interaction range.

At smaller densities, however, does $K_{\rho}^{\text {pert }}$ become poor. In particular, it does not reproduce the non-monotonic behavior of $K_{\rho}$ found in the HF approximation with a minimum as a function of density. Eventually, as $k_{\mathrm{F}} \rightarrow 0$ we conjecture that quantum wires approach the universality class of the Hubbard model with only on-site repulsion and that $K_{\rho} \rightarrow 1 / 2$ in that limit, though, unlike the Hubbard model, this limiting value should be approached from below as the particle density is lowered.

The non-monotonic dependence of $K_{\rho}$ on density predicted here should show up in any of the power lawd revealed by pseudo gaps in the density of states $\nu$. Examples include the current for tunneling into the end $\left(\nu(\omega) \sim \omega^{\left(1 / K_{\rho}-1\right) / 2}\right)$ or into the middle $\left(\nu(\omega) \sim \omega^{\left(K_{\rho}+1 / K_{\rho}-2\right) / 4}\right)$ of a single mode wire (assuming $K_{\sigma}=1$ ), and the current $I(V) \sim V^{1 / K_{\rho}}$ flowing through a single tunnel barrier along the wire at small voltages $V$. Experimental observation of this nonmonotonic dependence of the exponent would give direct experimental access to the microscopic range of the electronelectron interaction; the position and the height of the maximum in $K_{\rho}^{-1}$ both depend on $R$.

Our approach is less successful in estimating the spin sector TL parameter $v_{\sigma}$, at least when it differs considerably from $v_{\mathrm{F}}$. We have discussed perturbation theory and tried to obtain meaningful estimates for $v_{\sigma}$ from the HF spin density wave states. The resemblance to the antiferromagnetic Heisenberg spin chain, suggested by correlation function considerations, suggests that exchange coupling strengths, and therefore spin velocities, can be estimated by comparing the ground state energies of unpolarized and fully spin polarized electrons. None of these variants lead to results of the same quantitative reliability as those obtained from $K_{\rho}^{\mathrm{HF}}$. Conjecturing again a cross over into the universality class of the Hubbard model in the limit of $k_{\mathrm{F}} \rightarrow 0$ yields the prediction of a linear dependence of the relative spin velocity $v_{\sigma} / v_{\mathrm{F}} \propto k_{\mathrm{F}} / V_{0}$ on the particle density. $V_{0}$ is the zeroth Fourier component of the interaction $V\left(x-x^{\prime}\right)$.

It is important to kpgw the spin velocities for attempts to realize 'spintronic' devices where spins rather than charges are transported 26, using for example the Rashba spin precession mechanism 58 through quasi one-dimensional

constrictions 27. Here, in agreement with QMC estimates 37 , we have collected strong evidence that spin density excitations move at speeds considerably slower than the Fermi velocity, already in present day devices $\mathbb{3}$, where $k_{\mathrm{F}} d \approx$ 0.3 .

\section{Acknowledgement}

We would like to thank Ulrich Zülicke, Charles Creffield, and Hermann Grabert for valuable discussions. WH acknowledges the enjoyable hospitality of Indiana University at Bloomington, and also kind hospitalities of the University of Freiburg, and the King's College London where parts of this work have been carried out. Support has been received from the Deutsche Forschungsgemeinschaft (HA 2108/4-1), from the British EPSRC, and from the National Science Foundation under grant DMR0105947.

\section{APPENDIX A: HARTREE-FOCK THEORY}

To formulate the mean field theory we introduce single particle wave functions $\psi$ solving the Schrödinger equation

$$
\left\{-\frac{v_{\mathrm{F}}}{2 k_{\mathrm{F}}} \partial_{x}^{2}+\sum_{s^{\prime}} V_{s^{\prime}}^{\mathrm{H}}(x)\right\} \psi_{k s}(x)-\int_{0}^{L} \mathrm{~d} x^{\prime} V_{s}^{\mathrm{E}}\left(x, x^{\prime}\right) \psi_{k s}\left(x^{\prime}\right)=\varepsilon_{k s} \psi_{k s}(x)
$$

with the Hartree

$$
V_{s^{\prime}}^{\mathrm{H}}(x)=\frac{L}{2 \pi} \int_{0}^{L} \mathrm{~d} x^{\prime} V\left(x-x^{\prime}\right) \int_{-k_{\mathrm{F}}}^{k_{\mathrm{F}}} \mathrm{d} k\left|\psi_{k s^{\prime}}\left(x^{\prime}\right)\right|^{2}
$$

and the non-local exchange

$$
V_{s}^{\mathrm{E}}\left(x, x^{\prime}\right)=\frac{L}{2 \pi} V\left(x-x^{\prime}\right) \int_{-k_{\mathrm{F}}}^{k_{\mathrm{F}}} \mathrm{d} k \psi_{k s}^{*}\left(x^{\prime}\right) \psi_{k s}(x)
$$

potentials, which itself depend on $\psi$ and thus have to be obtained selfconsistently; $s=\uparrow, \downarrow$ are spin quantum numbers. Occupation of only the lowest subband is assumed together with periodic boundary conditions for the wire of length $L$. Parabolic dispersion for the kinetic energy in (A1) is described by a band mass $m=k_{\mathrm{F}} / v_{\mathrm{F}}$. The kinetic energy is not linearized. 
For some of our calculations, particularly those focussing on properties of the spin sector, cf. Section $\mathrm{V}$, we solved the HF equations (A1) directly in real space, using

$$
V(|x|)=\frac{e^{2}}{\epsilon}\left(\frac{1}{\sqrt{x^{2}+d^{2}}}-\frac{1}{\sqrt{x^{2}+d^{2}+4 R^{2}}}\right)
$$

in (A2, A3 and a lattice grid of at least 401 points. Any of the results for the charge sector can be obtained either using a real space basis as also, slightly more efficiently, in $k$-space, introduced now. Expanding

$$
\psi_{k s}(x)=\mathrm{e}^{\mathrm{i} k x} \sum_{j} u_{j, k, s} \mathrm{e}^{\mathrm{i} j 2 k_{\mathrm{F}} x}
$$

into Bloch waves, and similarly the periodic potentials (A2) and (A3), yields HF equations for the coefficients $u_{j, k, s}$

$$
\begin{aligned}
0= & {\left[\frac{1}{2}\left(2 j-\frac{k}{k_{\mathrm{F}}}\right)^{2}-\frac{\varepsilon_{k s}}{k_{\mathrm{F}} v_{\mathrm{F}}}\right] u_{j, k, s}+\frac{L}{2 k_{\mathrm{F}} \pi} \sum_{j^{\prime} j^{\prime \prime}} u_{j^{\prime \prime}, k, s} \int_{-k_{\mathrm{F}}}^{k_{\mathrm{F}}} \mathrm{d} k^{\prime} } \\
& \times\left\{\hat{V}\left(2\left(j-j^{\prime \prime}\right)\right) \sum_{s^{\prime}} u_{-j+j^{\prime}+j^{\prime \prime}, k^{\prime}, s^{\prime}}^{*} u_{j^{\prime}, k^{\prime}, s^{\prime}}-\hat{V}\left(2\left(j-j^{\prime}\right)-\frac{k}{k_{\mathrm{F}}}+\frac{k^{\prime}}{k_{\mathrm{F}}}\right) u_{-j+j^{\prime}+j^{\prime \prime}, k^{\prime}, s}^{*} u_{j^{\prime}, k^{\prime}, s}\right\} .
\end{aligned}
$$

For each $s= \pm 1$ and $k=-k_{\mathrm{F}} \ldots k_{\mathrm{F}}$ inside the Brillouin zone this is an eigenvalue equation for matrices indexed by the band indices $j$ which, however, inside the curly bracket, depends on the solution of (A6). Within the 'unrestricted'

HF scheme we allow for charge and spin density wave solutions breaking the symmetry of continuous translations and thereby lower the ground state energy. Solutions are found to show $4 k_{\mathrm{F}}$-periodic oscillations of the charge density $\varrho(x)=\varrho_{\uparrow}(x)+\varrho_{\downarrow}(x)$ where

$$
\varrho_{\uparrow}(x) \equiv \int_{-k_{\mathrm{F}}}^{k_{\mathrm{F}}} \mathrm{d} k\left|\psi_{k \uparrow}(x)\right|^{2}=\varrho_{\downarrow}\left(x+\frac{2 \pi}{4 k_{\mathrm{F}}}\right)
$$

We solved Eq. (A6) iteratively, starting with a sinusoidal spin density wave $u_{j, k, s}^{(0)}=\delta_{j, 0} / \sqrt{2}+s \delta_{|j|, 1} / 2$. The final solution always obeys $u_{j, k, \uparrow}=(-1)^{j} u_{j, k, \downarrow}$ which in view of (A7) yields $2 k_{\mathrm{F}}$-periodic modulations of the spin density $\varrho_{\uparrow}(x)-\varrho_{\downarrow}(x)$. A typical density modulation at stronger interaction is shown in Figure 1 .

The single particle energies $\varepsilon_{k s}$, obtained with (A6), determine the ground state energy

$$
\begin{aligned}
\frac{E_{0}}{L}=\sum_{s} \int_{-k_{\mathrm{F}}}^{k_{\mathrm{F}}} \frac{\mathrm{d} k}{2 \pi} \varepsilon_{k s} & -\frac{1}{2} \sum_{s s^{\prime}} \int_{-k_{\mathrm{F}}}^{k_{\mathrm{F}}} \frac{\mathrm{d} k}{2 \pi} \int_{0}^{L} \mathrm{~d} x V_{s^{\prime}}^{\mathrm{H}}(x)\left|\psi_{k s}(x)\right|^{2} \\
& +\sum_{s} \int_{-k_{\mathrm{F}}}^{k_{\mathrm{F}}} \frac{\mathrm{d} k}{2 \pi} \int \mathrm{d} x \int_{0}^{L} \mathrm{~d} x^{\prime} V_{s}^{\mathrm{E}}\left(x, x^{\prime}\right) \psi_{k s}^{*}(x) \psi_{k s}\left(x^{\prime}\right)
\end{aligned}
$$

Half of the interaction has to be subtracted to repair for its double counting in (A6). Differentiating $E_{0} / L$ twice w.r.t. $k_{\mathrm{F}}$ yields our estimate for $K_{\rho}$, according to (3).

Most of the results are obtained for $82 k$-points in the Brillouin zone (in some cases of very small densities we increased this number to 234). The Milne-rule, being accurate to 7 -th order in the spacing between $k$-points, is used for the $k$-integrations. We included $j=-3, \ldots, 3$ bands though in most cases $j=-2, \ldots, 2$ would have sufficed due to the rapid decay of the Coulomb interaction in $k$-space.

\section{APPENDIX B: HARTREE-FOCK FOR SUSCEPTIBILITIES}

In spin space we use a lattice representation $\psi\left(x=x_{i}\right) \rightarrow \psi(i)$ of the Hamiltonian. The $M \times M$ matrices $H_{i j}$, representing Eq. (A1), contain contributions from the kinetic energy $H_{i i}=2(M / \pi N)^{2} v_{\mathrm{F}} k_{\mathrm{F}}$ and $H_{i i \pm 1}=$ $-(M / \pi N)^{2} v_{\mathrm{F}} k_{\mathrm{F}}$, the (local) Hartree-term $H_{i i}=\sum_{k j s} \tilde{V}(|i-j| L / M)\left|\psi_{k s}(j)\right|^{2}$, and the (non-local) Fock-term $H_{i j}=$ $-\sum_{k} \tilde{V}(|i-j| L / M) \psi_{k s}(i) \psi_{k s}(j)$, the latter acting only on spin-s wave functions. Here, $\tilde{V}(x) \equiv \sum_{l=-\infty}^{\infty} V(x+l L)$ accounts for periodic boundary conditions $(V(x)$ is defined in (A4 $)$ and the real and normalized eigenvectors $\psi_{k s}(j)$ 
of $H_{i j}$ are indexed by their spin $s= \pm 1$ and momentum $-k_{\mathrm{F} s} \leq k \leq k_{\mathrm{F} s}=(1+s m) k_{\mathrm{F}}$ with $k$ being an integer multiple of $2 \pi / L$ and $m$ the magnetization per particle. The Hartree-Fock approximation to the ground state energy $E_{0}$ then is obtained as in Eq. (A8).

${ }^{1}$ S. Tomonaga, Prog. Theor. Phys. 5, 544 (1950).

2 J.M. Luttinger, J. Math. Phys. 4, 1154 (1963).

${ }^{3}$ F.D.M. Haldane, J. Phys. C 14, 2585 (1981); F.D.M. Haldane, Phys. Rev. Lett. 47, 1840 (1981).

${ }^{4}$ A.H. MacDonald, Phys. Rev. Lett. 64, 220 (1990); X.-G. Wen, Phys. Rev. Lett. 64, 2206 (1990); Phys. Rev. B 41, 12838 (1990); Phys. Rev. B 43, 11025 (1991); Int. J. Mod. Phys. B 6, 1711 (1992); U. Zülicke and A.H. MacDonald, Phys. Rev. B 54, 16813 (1996).

${ }^{5}$ C.L. Kane and M.P.A. Fisher, Phys. Rev. B 46, 7268 (1992); A. Furusaki and N. Nagaosa, Phys. Rev. B 47, 4631 (1993).

${ }^{6}$ S.J. Tans, M.H. Devoret, H. Dai, A. Thess, R.E. Smalley, L.J. Geerligs, and C. Dekker, Nature 386, 474 (1997); M. Bockrath, D.H. Cobden, J. Lu, A.G. Rinzler, G. Andrew, R.E. Smalley, L. Balents, and P.L. McEuen, Nature 397, 598 (1999); Z. Yao, H.W.J. Postma, L. Balents, and C. Dekker, Nature 402, 273 (1999).

${ }^{7}$ R. Egger and A.O. Gogolin, Phys. Rev. Lett. 79, 5082 (1997); C. Kane, L. Balents, and M.P.A. Fisher, ibid. 79, 5086 (1997).

${ }^{8}$ S. Tarucha, T. Honda, and T. Saku, Solid State Comm. 94, 413 (1995).

${ }^{9}$ A. Yacoby, H.L. Stormer, N.S. Wingreen, L.N. Pfeiffer, K.W. Baldwin, and K.W. West, Phys. Rev. Lett. 77, 4612 (1996); O.M. Auslaender, A. Yacoby, R. de Picciotto, K.W. Baldwin, L.N. Pfeiffer, and K.W. West, Phys. Rev. Lett. 84, 1764 (2000); M. Rother, W. Wegscheider, R.A. Deutschmann, M. Bichler, and G. Abstreiter, Physica E 6, 551 (2000).

${ }^{10}$ W. Apel and T.M. Rice, Phys. Rev. B 26, 7063 (1982).

${ }^{11}$ D.L. Maslov and M. Stone, Phys. Rev. B 52, R5539 (1995); V.V. Ponomarenko, Phys. Rev. B 52, R8666 (1995); I. Safi and H.J. Schulz, Phys. Rev. B 52, R17040 (1995); R. Egger and H. Grabert, Phys. Rev. Lett. 77, 538 (1996); Phys. Rev. B 58, 10761 (1998); F. Kassubek, Diploma Thesis, Univ. of Freiburg (1997).

${ }^{12}$ V. Meden, P. Schmitteckert, and N. Shannon, Phys. Rev. B 57, 8878 (1998).

13 A.M. Finkel'shteǐn, Pis'ma Zh. Eksp. Teor. Fiz. 25, 83 (1977) [JEPT Lett. 25, 73 (1977)].

${ }^{14}$ I. Affleck, D. Gepner, H.J. Schulz, and T. Ziman, J. Phys. A 22, 511 (1989).

${ }^{15}$ H.J. Schulz, Phys. Rev. Lett. 71, 1864 (1993).

${ }^{16}$ M. Fabrizio, A.O. Gogolin, and S. Scheidl, Phys. Rev. Lett. 72, 2235 (1994).

${ }^{17}$ For a review see e.g. J. Voit, Rep. Prog. Phys. 58, 977 (1995).

${ }^{18}$ Galilei invariance ensures that no physical property is affected by $\nu(k) \rightarrow \nu(k+q)$ for any $q$; $\nu(k)$ are density modulations of wave vector $k$ and $\nu=\rho, \sigma$. For the TL follows that $K_{\rho} v_{\rho}$ does not change with interactions depending on the distance.

19 B. Tanatar and D.M. Ceperley, Phys. Rev. B 39, 5005 (1989); S.T. Chui and B. Tanatar, Phys. Rev. Lett. 74, 458 (1995).

${ }^{20}$ K. Schönhammer, V. Meden, W. Metzner, U. Schollwöck, and O. Gunnarsson, Phys. Rev. B 61, 4393 (2000).

${ }^{21}$ Q.P. Li, S. Das Sarma, and R. Joynt, Phys. Rev. B 45, 13713 (1992).

${ }^{22}$ As demonstrated after careful summation of the series : I.E. Dzyaloshinskiu and A.I. Larkin, Sov. Phys. JETP, 38, 202 (1974).

23 J. Sólyom, Advances in Physics 28, 201 (1979).

${ }^{24}$ A. Luther and V.J. Emery, Phys. Rev. Lett. 33, 589 (1974). A. Luther, Phys. Rev. B 15, 403 (1977).

${ }^{25}$ G.D. Mahan, Many-Particle Physics, Plenum (1990).

${ }^{26}$ G.A. Prinz, Physics Today 48, No. 4, 58 (1995).

${ }^{27}$ K. Tsukagoshi, B.W. Alphenaar, and H. Ago, Nature 401, 572 (1999).

${ }^{28}$ L. Balents and R. Egger, Phys. Rev. Lett. 85, 3464 (2000); cond-mat/0012192

${ }^{29}$ W. Häusler, Phys. Rev. B 63, 121310 (2001).

${ }^{30}$ A. Pinczuk and G. Abstreiter in Light Scattering in Solids, ed. by M. Cardona and G. Güntherodt, Springer (1989); D.W. Wang, A.J. Millis, and S. Das Sarma, Phys. Rev. Lett. 85, 4570 (2000).

31 A.R. Goñi, A. Pinczuk, J.S. Weiner, J.M. Calleja, B.S. Dennis, L.N. Pfeiffer, and K.W. West, Phys. Rev. Lett. 67, 3298 (1991); G. Biese, C. Schüller, K. Keller, C. Steinebach, and D. Heitmann, Phys. Rev. B 53, 9565 (1996); ibid. 54, R17304 (1996).

${ }^{32}$ N. Shibata, A. Tsvelik, K. Ueda, Phys. Rev. B 56, 330 (1997).

${ }^{33}$ H.J. Schulz, Phys. Rev. Lett. 64, 2831 (1990); H. Frahm and V.E. Korepin, Phys. Rev. B 42, 10554 (1990).

${ }^{34}$ N. Kawakami and S.-K. Yang, Phys. Rev. Lett. 65, 2309 (1990); S. Sarkar, J. Phys. A: Math. Gen. 23, L409 (1990).

${ }^{35}$ M. Ogata, M.U. Luchini, S. Sorella, and F.F. Assaad, Phys. Rev. Lett. 66, 2388 (1991).

${ }^{36}$ N. Kawakami and S.-K. Yang, Phys. Rev. Lett. 67, 2493 (1991).

${ }^{37}$ C.E. Creffield, W. Häusler, and A.H. MacDonald, Europhys. Lett. 53, 221 (2001). 
${ }^{38}$ D. Yoshioka and P.A. Lee, Phys. Rev. B 27, 4986 (1983).

39 Throughout this work by 'perturbation theory' we mean the expressions obtained in lowest order.

${ }^{40}$ K. Byczuk and T. Dietl, Phys. Rev. B 60, 1507 (1999).

${ }^{41}$ G.Y. Hu and R.F. O'Connell, Phys. Rev. B 42, 1290 (1990).

42 J. Friesen, A. Bergensen, J. Phys. C 13, 6627 (1980).

${ }^{43}$ D.C. Mattis, The Many-Body Problem, An Encyclopedia of Exactly Solved Models in One Dimension, World Scientific, Singapore (1993).

${ }^{44}$ P. Schlottmann, J. Phys. C : Cond. Matt. 6, 1359 (1994).

${ }^{45}$ H.J. Schulz, Int. J. Mod. Phys. 5, 57 (1991).

${ }^{46}$ A.W. Overhauser, Physics 1, 307 (1965).

${ }^{47}$ C.F. Coll, Phys. Rev. B 9, 2150 (1974).

${ }^{48}$ H. Frahm and V.E. Korepin, Phys. Rev. B 42, 10553 (1990).

${ }^{49}$ H.J. Schulz in Correlated Electron Systems, ed. by V.J. Emery, World Scientific (1993), p. 199;

${ }^{50}$ See for example L. Zheng and A.H. MacDonald, Phys. Rev. B 48, 8203 (1993).

${ }^{51}$ E. Lieb and D. Mattis, Phys. Rev. 125, 164 (1962).

52 J.M.P. Carmelo, P. Horsch, D.K. Campbell, and A.H. Castro Neto, Phys. Rev. B 48, 4200 (1993).

${ }^{53}$ W. Häusler, Advances in Solid State Physics 34, 171 (1994); Annalen der Physik 5, 401 (1996); Z. Phys. B 99, 551 (1996); J.H. Jefferson and W. Häusler, Phys. Rev. B 54, 4936 (1996).

${ }^{54}$ A.M. Polyakov und P.B. Wiegmann, Phys. Lett. B 131, 121 (1983); P.B. Wiegmann, Phys. Lett. B 141,217 (1984), ibid. 142, 173 (1984); E. Witten, Commun. Math. Phys. 92, 455 (1984).

${ }^{55}$ L. Hulthén, Arkiv Mat. Astron. Fysik 26A, No. 11 (1938).

${ }^{56}$ L. Calmels and A. Gold, Europhys. Lett. 39, 539 (1997).

${ }^{57}$ See for example K.S. Singwi and M.P. Tosi, Solid State Physics 36, 177 (1981).

${ }^{58}$ B. Datta and S. Das, Appl. Phys. Lett. 56, 665 (1990). 


\section{Figures}

FIG. 1.

Figure 1: Charge densities $n_{\uparrow}(x)$ (solid) and $n_{\downarrow}(x)$ (dashed) as a function of position along the wire $x$ in units of the mean electron spacing for $k_{\mathrm{F}} d=0.15$ and $R / d=5.66$. We argue that these charge densities in the broken symmetry Hartree-Fock states are a good approximation to typical configurations in the fluctuating one-dimensional electron liquid that does not have broken translational symmetry.

FIG. 2.

Figure 2: Fourier transforms of three different forms of microscopic electron-electron interactions, $\hat{V}(k)=V(k) / v_{\mathrm{F}}$, as described in the text. Solid: the form (国) we use in the present work, dashed: the 2D heterostructures model, dash dotted: the 3D cylindrical case intended for cleaved edge overgrowth systems

FIG. 3.

Figure 3: Ground state energy densities $e_{0}\left(k_{\mathrm{F}}\right)=\left(E_{0} / L\right) /\left(k_{\mathrm{F}}^{3} / m\right)$ for $R / d=5.66$ (a) and for $R / d=35.36$ (b) in $\mathrm{HF}$ (solid). The perturbative estimate (Eq. (9), long-dashed) establishes an upper bound while the harmonic chain estimates, both, omitting (Eq. (7), dotted) or including quantum fluctuations (Eq. (6), dashed) both establish lower bounds to the true ground state energy.

FIG. 4.

Figure 4: $1 / K_{\rho}$ versus $k_{\mathrm{F}} d$ for $R / d=5.66$ (a) and for $R / d=35.36$ (b). The same approximations are included as in Figure 3, together with the commonly used formula (1), dash-dotted.

FIG. 5.

Figure 5: $1 / K_{\rho}$ versus $R / d$ for $k_{\mathrm{F}} d=0.15$ in HF (solid), perturbation theory (Eq. (12), long-dashed), and for the harmonic chain (Eq. (7), dashed) and (Eq. (6), dotted).

FIG. 6.

Figure 6: Amplitudes for the $2 k_{\mathrm{F}}$ and $4 k_{\mathrm{F}}$-periodic components of the charge density modulations $\rho_{1}$ and $\rho_{2}$ versus $k_{\mathrm{F}} d$ for $R / d=35.36$ in units of $\rho_{0}=2 k_{\mathrm{F}} / \pi$.

FIG. 7.

Figure 7: Charge densities $n_{\uparrow}(x)$ (solid) and $n_{\downarrow}(x)$ (dashed) along the wire $x$ in units of the mean electron spacing, as in Figure 1 but for finite magnetization $m=1 / 6$ per spin.

FIG. 8.

Figure 8: Estimates to spin velocities $v_{\sigma} / v_{\mathrm{F}}$, based on the selfconsistent Hartree-Fock solution : 'HF', on perturbation theory, Eq. (19): 'pert', and on the comparison with the antiferromagnetic Heisenberg model, Eq. (21) : 'J'. 


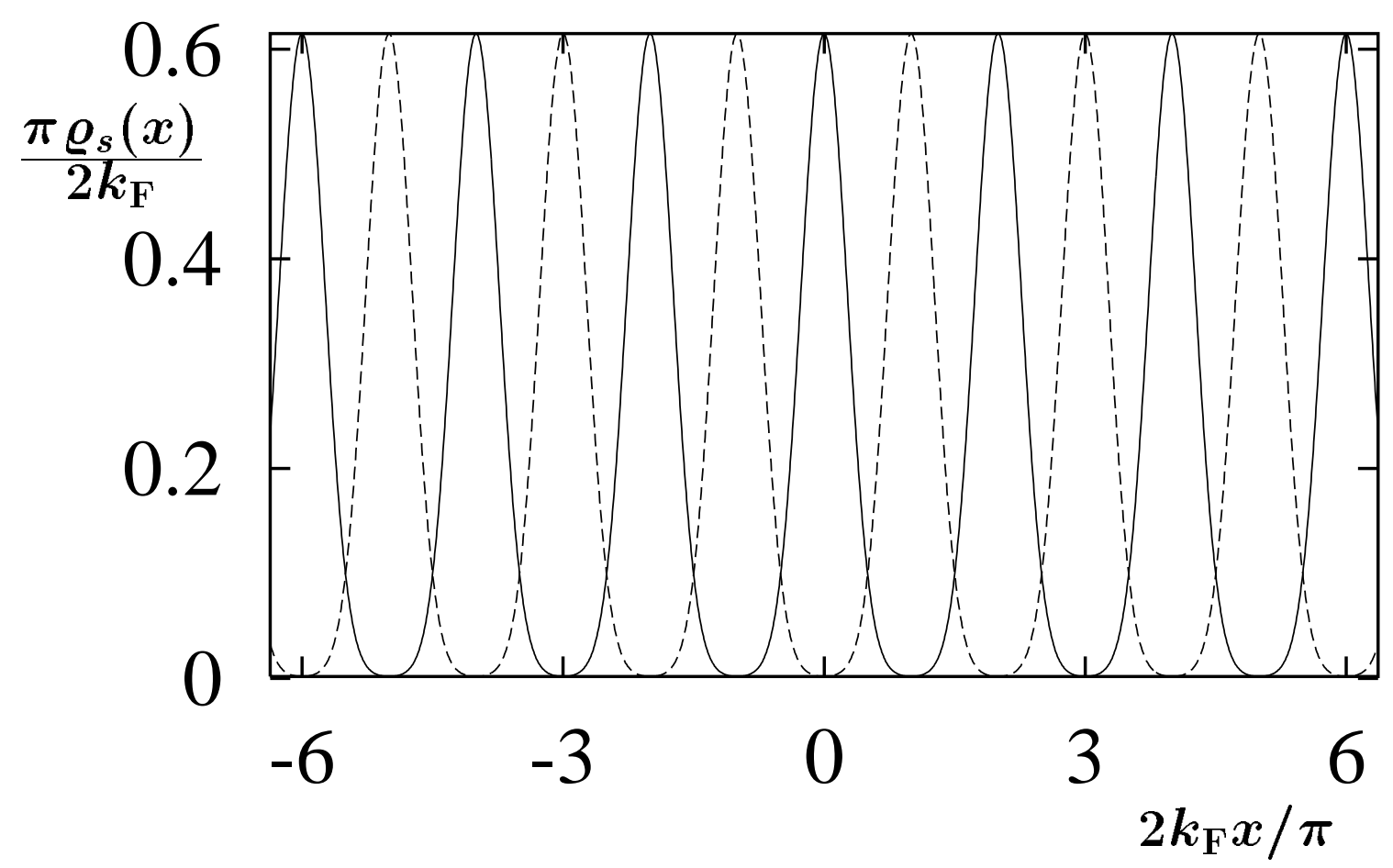

FIGURE 1 


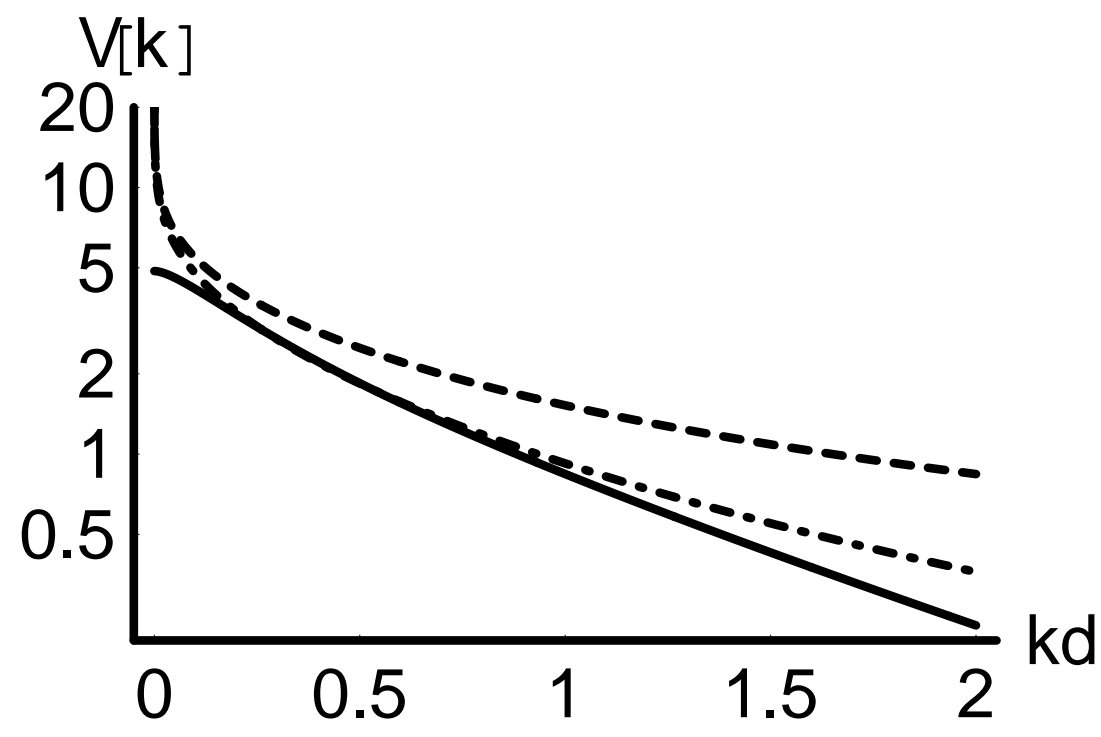

FIGURE 2 


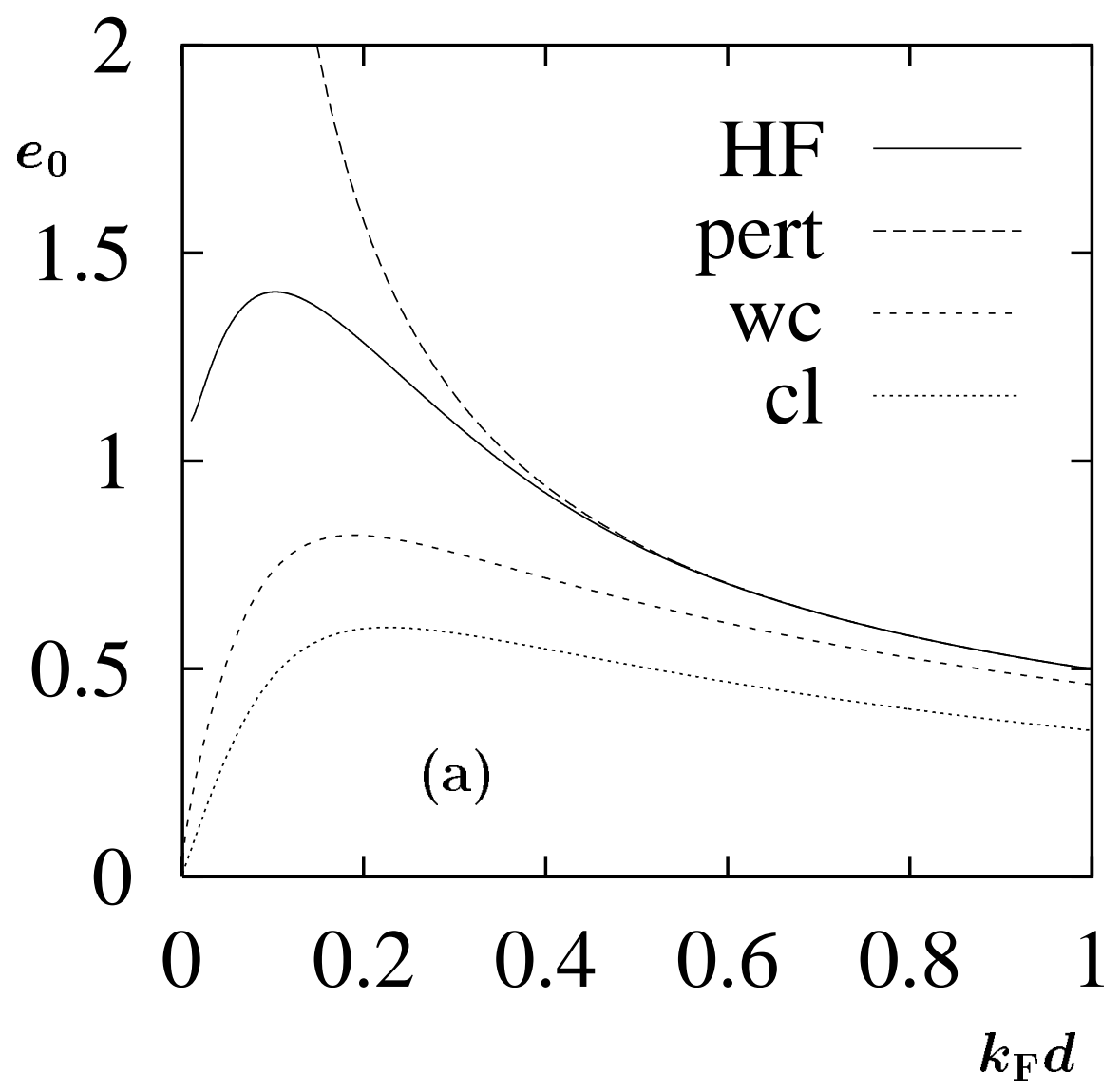

FIGURE 3a 


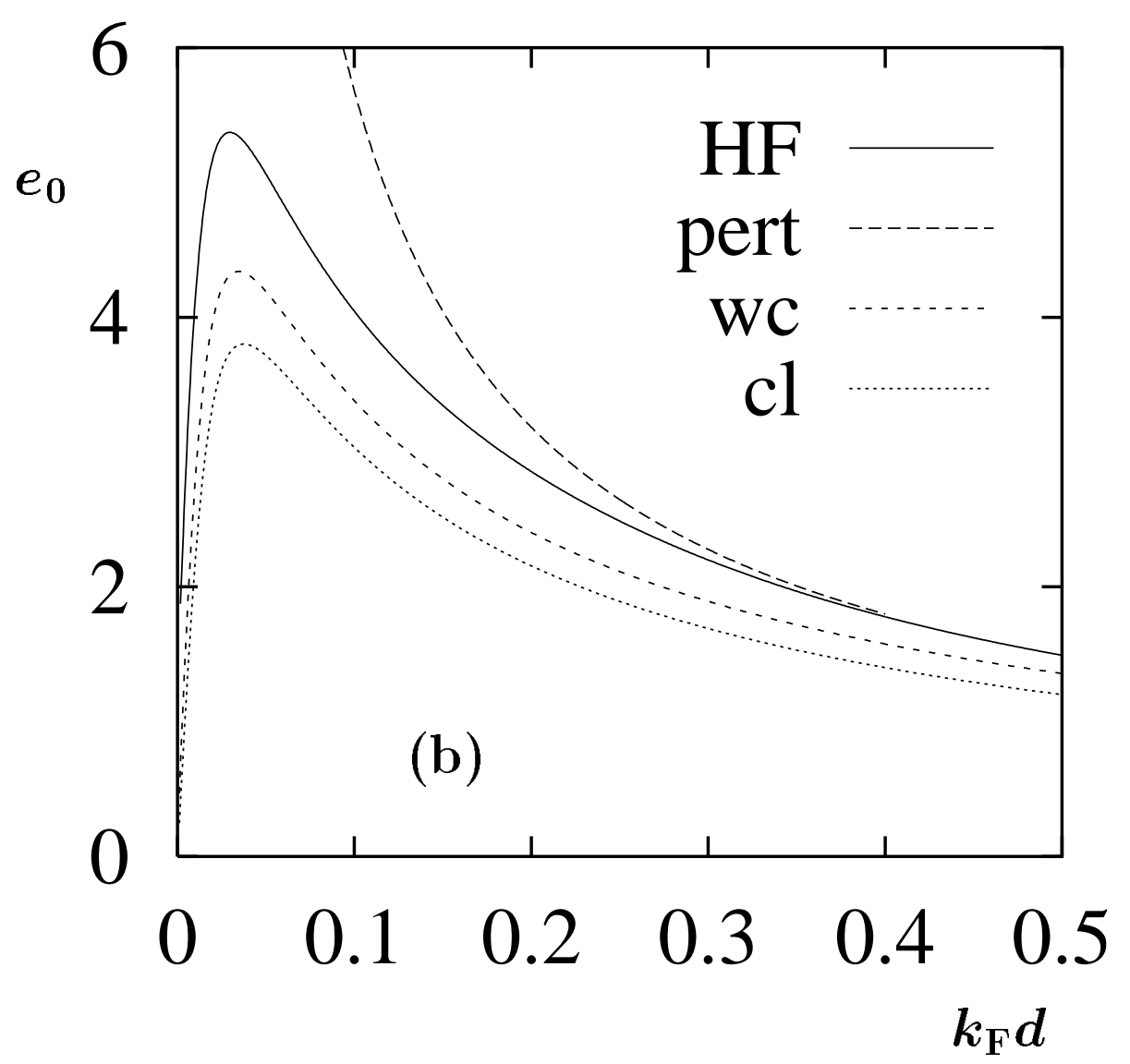

FIGURE $3 \mathrm{~b}$ 


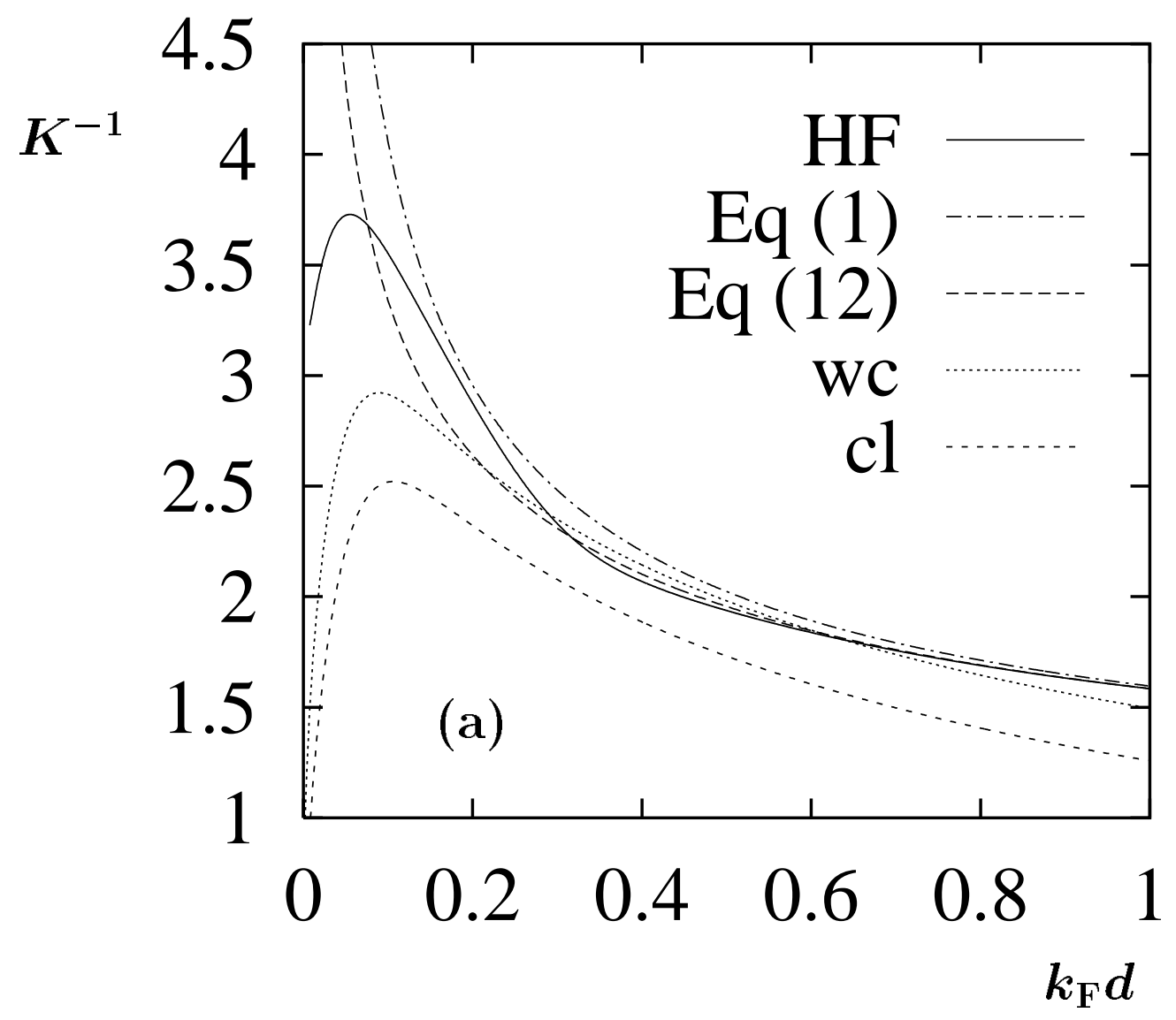

FIGURE $4 a$ 


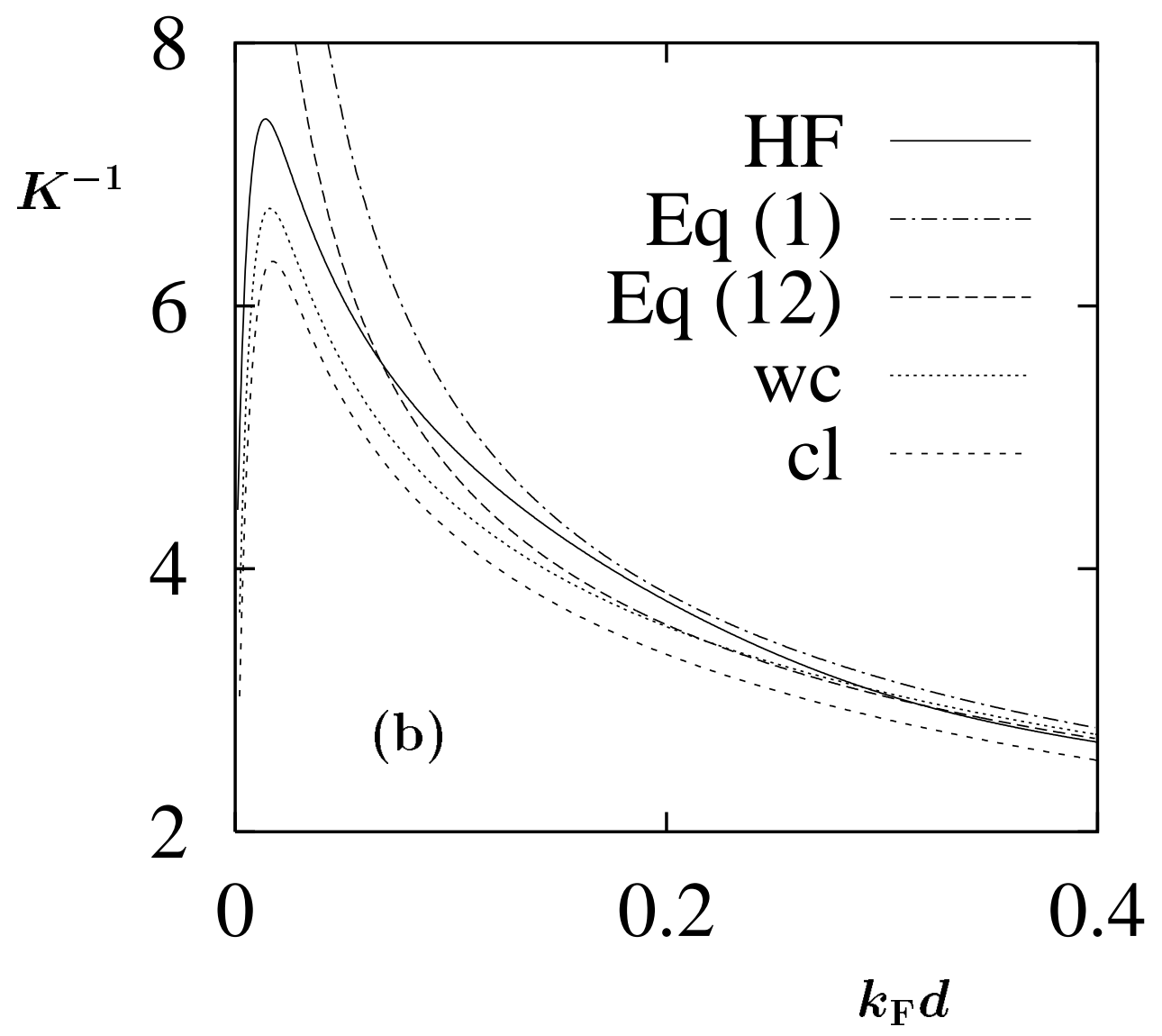

FIGURE $4 b$ 


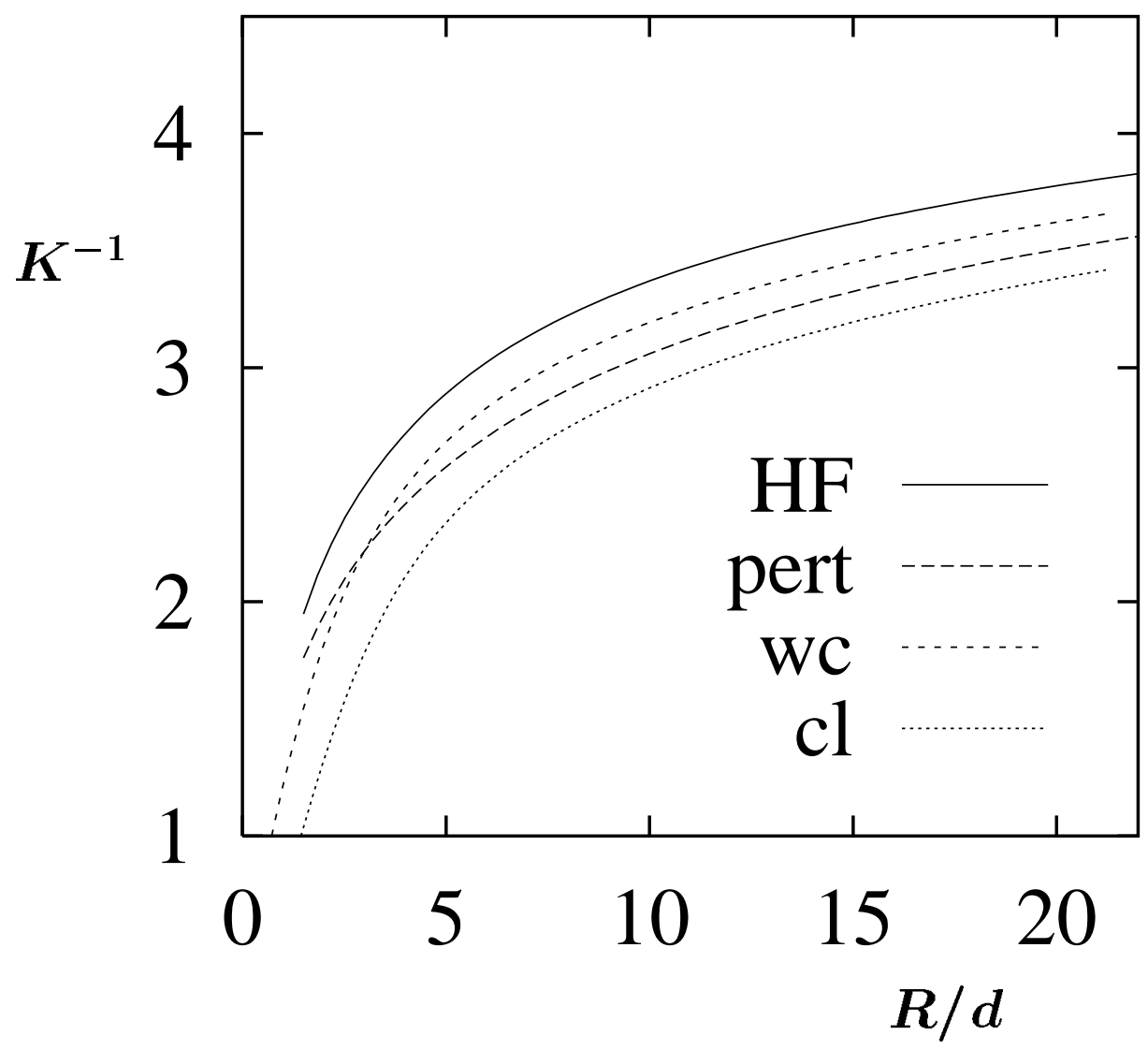

FIGURE 5 


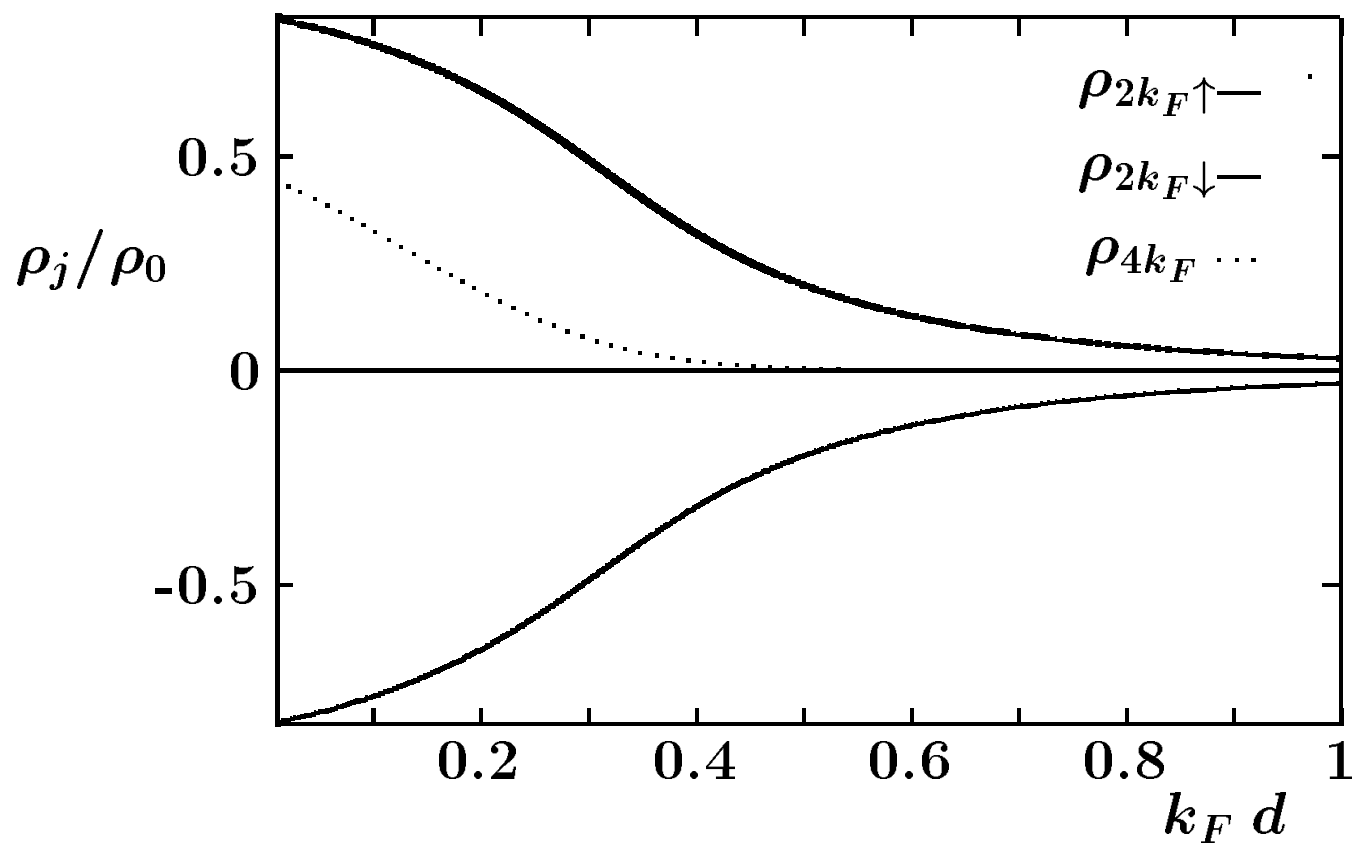

FIGURE 6 


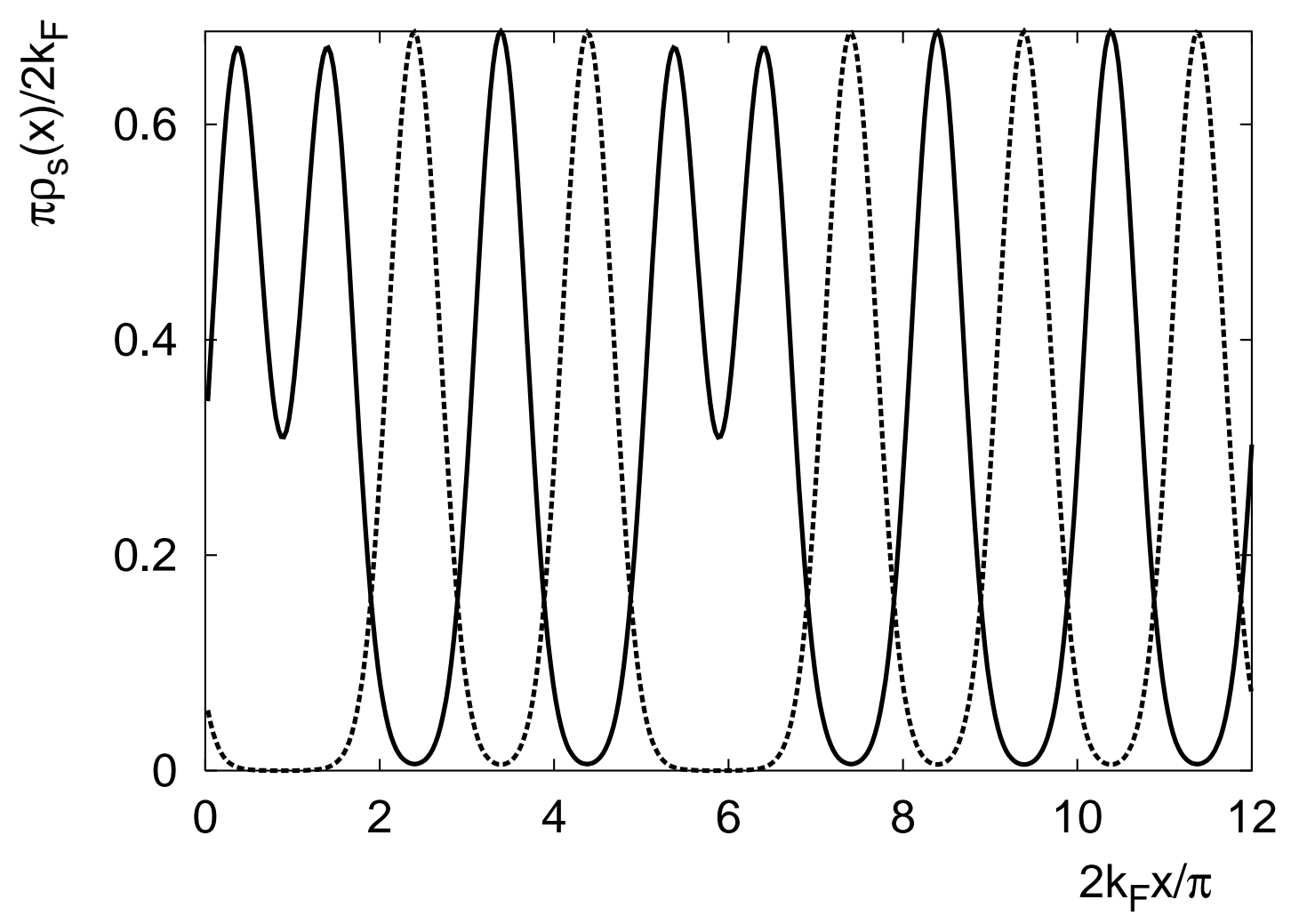

FIGURE 7 


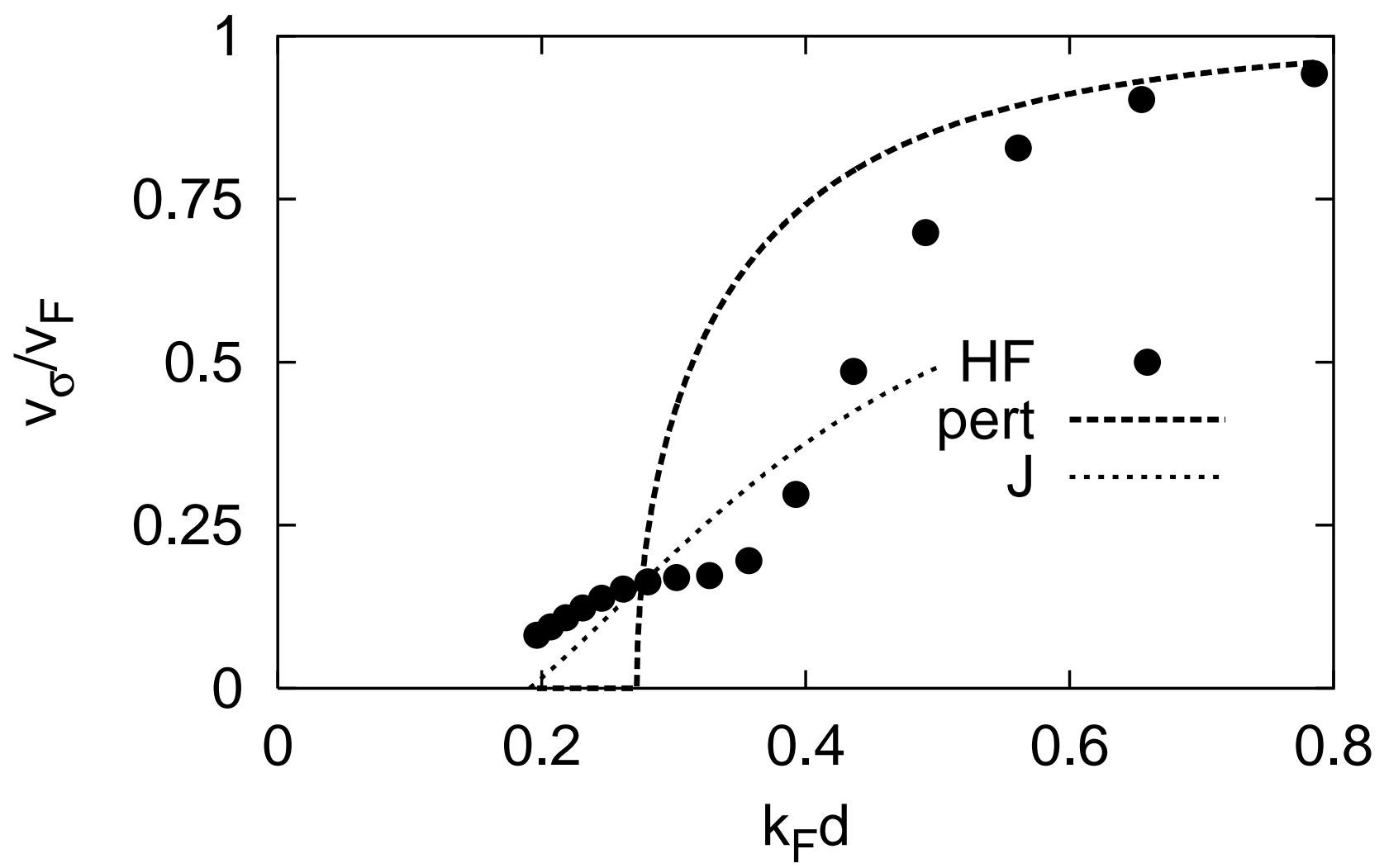

FIGURE 8 